\section{Immunotherapy of hepatocellular carcinoma}

\author{
Bruno Sangro ${ }^{* 1}$, Daniel Palmer² \& Ignacio Melero 3
}

\section{Practice points}

- Dendritic cell vaccination in hepatocellular carcinoma (HCC) patients is feasible and safe, and there is evidence of immune responses following vaccination but clinical efficacy is still not proved.

- Glypican-3 is overexpressed in up to $80 \%$ of HCC tumors and a vaccination strategy using GPC3derived epitopes has shown to produce some objective tumor responses in an early trial.

- Ongoing research projects are analyzing the peptidome of HCC patients to develop a universal vaccine comprising multiple tumor-associated peptides naturally presented in human tumors.

- IFN-a, the only immunostimulatory cytokine tested in HCC, is not effective in reducing postoperative recurrence of early $\mathrm{HCC}$ or prolonging survival among patients with inoperable tumors.

- Pexa-Vec, an armed oncolytic poxvirus that carries the human GM-CSF gene, was able to produce objective tumor responses in early trials but has failed to prolong survival among patients with advanced HCC who had failed sorafenib.

- Tremelimumab, a human monoclonal antibody that binds to CTLA-4, has recently shown encouraging signs of antitumor and antiviral activity in HCC patients with hepatitis $C$ virus infection. This has paved the way for other immune checkpoint inhibitors like the PD-1-blocking nivolumab that is under extensive clinical testing.

- There is a wide consensus that combination immunotherapy strategies should be developed including the combination of different checkpoint inhibitors or the combination of these agents with vaccines, adoptive T-cell therapy or conventional therapies.

SUMMARY Newer immunotherapy agents may break the barrier that tumors create to evade the attack from the immune system. Dendritic cell vaccination has shown encouraging clinical activity and a favorable safety profile in advanced tumor stages. However, optimal cell maturation status, choice of tumor antigens and route of administration have not been established. Single or multiple peptides derived from tumor-associated antigens may also be used for cancer vaccination. Intratumoral delivery of oncolytic viruses expressing immunostimulating cytokines like GM-CSF have produced stimulating clinical results that need further verification. But it is probably T-cell checkpoint modulation with monoclonal antibodies that has attracted the highest expectations. Promising activity has been reported for tremelimumab, a CTLA-4 inhibitor, and a clinical trial testing the PD-1 antibody nivolumab is underway. Future progress will probably come from a better understanding of the mechanisms of cancer-related immunosuppression, improvement in agents and strategies and combination of the available therapeutic tools.

\footnotetext{
'Liver Unit, Clínica Universidad de Navarra, and Centro de Investigación Biomédica en Red de Enfermedades Hepáticas y Digestivas (CIBEREHD); Avda. Pio XII 36. 31008-Pamplona, Spain

${ }^{2}$ The Department of Molecular \& Clinical Cancer Medicine, University of Liverpool, Liverpool, L69 3GA, UK

${ }^{3}$ Departments of Oncology \& Immunology, Centro de Investigación Médica Aplicada y Clínica Universidad de Navarra. Avda. Pio XII, 55. 31008-Pamplona, Spain

*Author for correspondence: Tel.: +34 948255 400; Fax: +34 948296 500; bsangro@unav.es
}

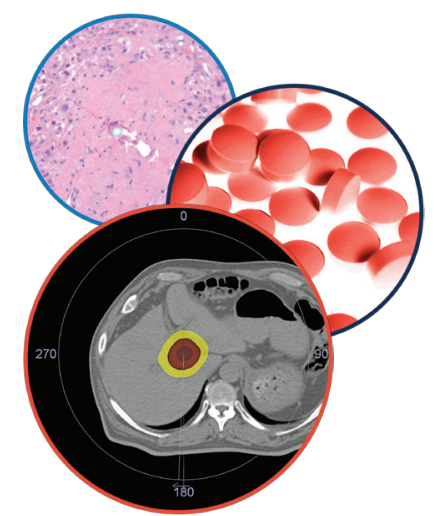

Hepatic Oncology

\section{KEYWORDS}

- cell therapy • dendritic cells • immunotherapy - monoclonal antibodies - NK cells • tremelimumab 
Cancer growth exemplifies a failure of the immune system. The proposal of cancer immunosurveillance and immunoediting [1] and other theories that may help explain the complex phenomena of immune escape by tumor cells [2] are beyond the scope of this review. Restoration and re-inforcement of the effectiveness of the immune system should therefore have a negative effect on cancer persistence and growth. After decades of defeats, immunotherapy has been recently integrated into the systemic therapy of melanoma and prostate cancer. Eyes turn now to other malignancies that may become amenable to immune modulation including hepatocellular carcinoma (HCC). With only one systemic therapy (sorafenib) currently approved for advanced HCC, we are in great need of new agents that may help improve the prognosis of this still dreadful tumor, alone or in combination.

HCC possesses characteristics that render it a potential target for immunotherapeutic manipulation. There is an active recruitment of lymphocytes, which have specific mechanisms to recognize and bind to tumor endothelium and infiltrate tumor tissue, suggesting a potential for cytotoxic effector cell activation [3] and there is a correlation between the density of lymphocytic infiltrates in HCC lesions and prognosis [4]. In addition, tumor infiltrating lymphocytes derived from HCC and then expanded ex vivo with IL-2 have the ability to lyse autologous tumor cells [5]. However, tumor infiltrating lymphocytes in HCC are only partially activated, proliferate only at very low levels and fail to kill tumor cells unless activated by IL-2 in vitro. The fact that HCC develops in immunocompetent hosts indicates a failure to mount an adequate antitumor immune response naturally. Tumors evade immune detection by generating an immune-inhibitory microenvironment through diverse mechanisms that include but are not limited to the secretion of immuno-inhibitory cytokines such as IL-8 [6], downregulation of the CD95 receptor and lymphocytes killing through expression of Fas ligand [7] and TRAIL [8], expression of ligands for T-cell inhibition such as PD-L1 [9,10], recruitment or induction of suppressive immune cells including regulatory $\mathrm{T}$ cells [11,12], MDSC [13] and invariant NKT cells [14].

Immune therapies of cancer can be classified based on the role of the immune system in the therapeutic response. Passive or adoptive immunotherapy is based on administration of antitumor antibodies or transfer of tumor reactive lymphocytes, while active immunotherapy aims at eliciting new specific immune response against tumor antigens (cancer vaccines) or at amplifying an existing although insufficient antitumor immune response by administering nonspecific proinflammatory molecules or adjuvants. Such cancer vaccines can be based on cells (dendritic cells [DC]), RNA or tumor-specific peptides.

On the other hand, immunotherapy can be classified from a product perspective in cell-based and non-cell-based strategies. The latter include agents such as cytokines, inductors of tumor cell immunogenic death, antibodies or biomolecules that signal through co-stimulatory molecules or block co-inhibitory receptors, and agents that cause depletion or inactivation of regulatory $\mathrm{T}$ cells or impact the function of myeloid-derived suppressor cells inside the tumor. Chemotherapeutic agents that may cause partial or specific immune cell depletion (i.e., gemcitabine depleting myeloid suppressor cells or cyclophosphamide depleting $\mathrm{T}$ regulatory cells) or those small molecules that influence metabolic pathways related to immunosupresive mechanisms (i.e., arginase, indoleamine dioxygenase or NO synthetase) will not be considered in this review.

Cell-based immunotherapy

A number of cell-based immunotherapy strategies have entered clinical trials or are in preclinical development for HCC including DC, cytokine-induced killer cells, NK cells and genetically modified T cells (Figure 1).

\section{- Dendritic cells}

The activation of tumor-specific cytotoxic $\mathrm{T}$ cells requires three synergistic signals: the presentation of tumor antigen by antigen-presenting cells to specific T-helper cells; the interaction between costimulatory factors (such as B7.1 and CD28 ligand); and the secretion of immunostimulatory cytokines (such as IL-2 and IL-12) by activated T-helper cells and antigenpresenting cells as well. Certain DC subtypes may also cross-present antigen directly to $\mathrm{CD} 8^{+}$ cytotoxic $\mathrm{T}$ cells through processing of exogenous antigen via the MHC class I pathway. DCs are the most potent professional antigenpresenting cells. They can capture, process and present antigens to naive $\mathrm{T}$ cells, stimulating their proliferation and activation. They provide 


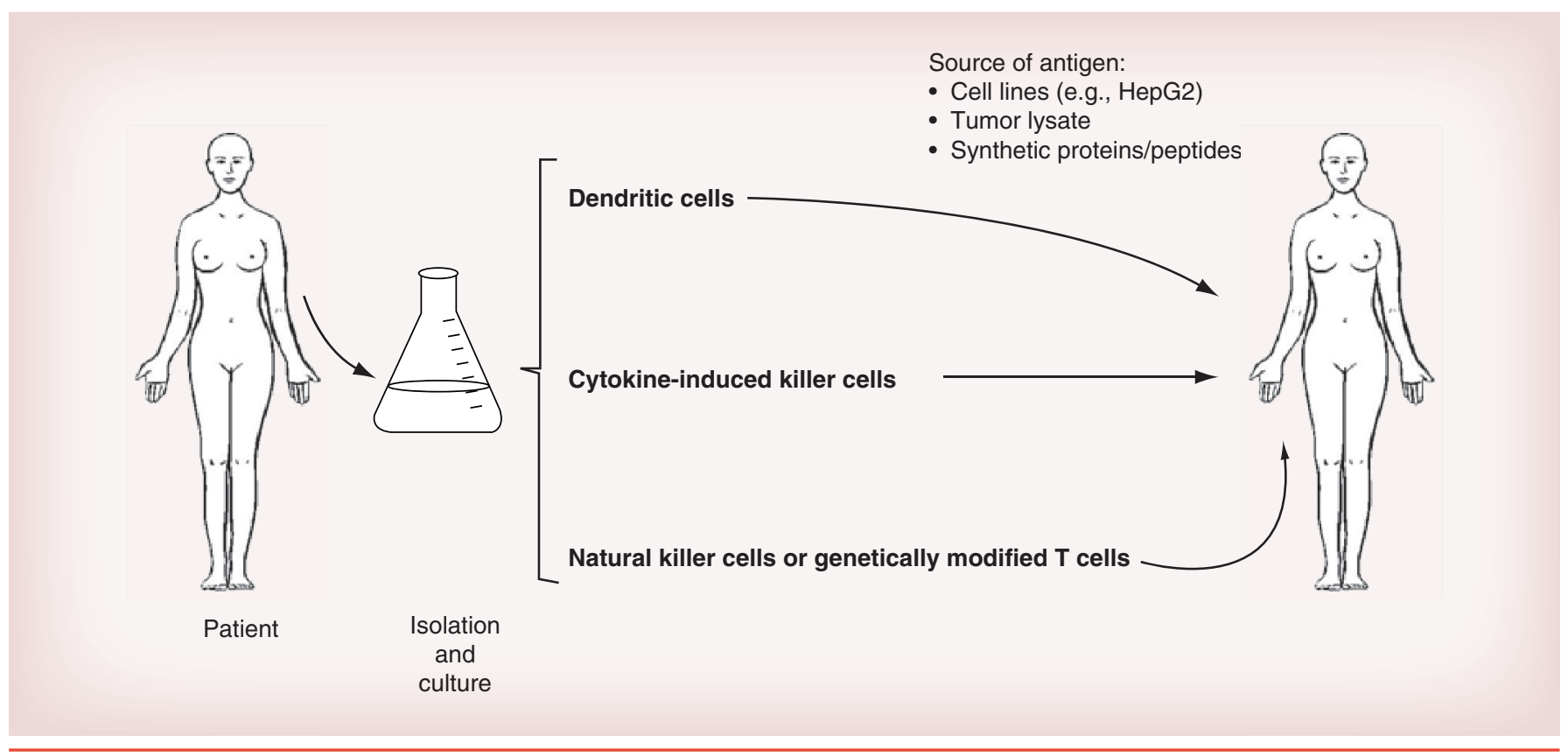

Figure 1. A summary of strategies used in cell-based immunotherapy of hepatocellular carcinoma.

the optimum costimulatory environment, with high levels of MHC I and II, costimulatory molecules (CD40, B7), adhesion molecules (intercellular cell adhesion molecule 1) and stimulatory cytokines (IL-12, IFN- $\alpha$ ) to evoke an immunostimulatory signal against that antigen.

However, DC function in HCC is suppressed as a result of the production of local factors within the tumor microenvironment, including IL-10, IL-6. M-CSF and VEGF, and this leads to a failure of DCs to induce antitumor immune responses through impaired differentiation, maturation and function and indeed, immature DCs may contribute to the immunosuppressive tumor microenvironment through a lack of appropriate costimulatory signals resulting in deletion of antigen-specific $\mathrm{T}$ cells and expansion of regulatory $\mathrm{T}$ cells $[15,16]$. Recent data have indicated that HCC may also induce a subset of $\mathrm{CD} 14^{+}$regulatory DC that further contributes to immunosuppression through production of IL-10 and IDO, which is dependent on CTLA4 expression on the DC and which suggests a potential additional mechanism of action for anti-CTLA4 antibody therapy [17]. This underpins the rationale for activating DCs in vitro to overcome tumor-associated immunosuppression and re-infusing them into patients to stimulate antitumor immunity. The use of DCs as a platform for therapeutic vaccines is facilitated by the ability to harvest them from peripheral blood in sufficient numbers, and to manipulate them ex vivo to present antigens of interest [18].

DC-based immunotherapy has been tested in clinical trials in melanoma, prostate cancer and renal cell cancer, as well as in HCC [19-24]. Safety has been demonstrated with variable efficacy. Based on a positive randomized Phase III trial in 512 patients with metastatic castrate-resistant prostate cancer, sipuleucel-T (Provenge, Dendreon) an autologous DC vaccine pulsed ex vivo with a fusion protein comprising a prostate cancer antigen (prostatic acid phosphatase) and GM-CSF has recently become the first US FDA-approved commercial cellular cancer vaccine, demonstrating that this technology can be broadly applied across multicenter, multinational clinical trials and as a standard of care [25].

A number of early phase trials of DC vaccination have been conducted in the setting of advanced HCC largely using autologous DC derived from PBMC and pulsed ex vivo with one or a number of tumor antigens. The largest study reported clinical activity of an autologous DC vaccine pulsed with the lysate of a liver cancer cell line, HepG2, as a source of potential tumor antigens, which include $\alpha$-fetoprotein (AFP) with evidence for the generation of antigenspecific immune responses. Immune responses against AFP and against the vaccine could be 
detected using ELIspot as a functional measure of the frequency of $\mathrm{T}$ cells that produce the Th1 immunostimulatory cytokine, IFN- $\gamma$, when stimulated by a specific antigen. In some cases, this was associated with a significant fall in serum AFP suggesting the generation of effective immune responses against the tumor [26]. A further trial randomized 30 patients with advanced HCC (and advanced underlying liver disease, mostly Child-Pugh B) to receive a similar HepG2-pulsed DC vaccine or supportive care. Again, vaccination was well tolerated with evidence of clinical and immunological responses. Survival was numerically longer in patients receiving vaccination (median 7 vs 4 months) but the trial is too small to draw meaningful conclusions from this [27]. A trial using DC loaded with autologous tumor lysate and using two different schedules of administration has similarly reported safety, reassuringly with no evidence of autoimmunity, with some evidence of efficacy and a suggestion that pulsed vaccination followed by monthly boosters may be superior to five pulsed vaccinations alone, although this was not a randomized comparison [23]. A study using immature DCs pulsed with human leukocyte antigen (HLA)-A0201restricted AFP peptides reported the induction of AFP-specific CD8 ${ }^{+}$T-cell responses, though no clinical responses were seen [22]. Subsequent analysis also demonstrated that DC vaccination could reduce the proportion of regulatory $\mathrm{T}$ cells and enhance CD $56^{+} / \mathrm{CD} 16^{+/-} \mathrm{NK}$ cells, which have the potential to kill tumor cells with low-level MHC expression that might otherwise escape immune recognition [28].

These studies illustrate several key points in the development of cellular immunotherapy for HCC. First, it is feasible to generate autologous DCs from patients even with advanced malignancy and coexistent chronic liver disease. These DCs can be antigen-loaded ex vivo and matured using the appropriate cytokine cocktail prior to re-infusion. Second, DC vaccination appears to be safe and tolerated well with no significant toxicity and, in some cases despite loading with multiple antigens from whole cell lysates, no evidence of autoimmunity. And third, there is evidence of immune responses following vaccination. However, there remain several unanswered questions in the further development of DC vaccines for HCC. These include the optimal maturation status of the DC, the choice of tumor antigens and the route of administration of the vaccine. Evidence suggests that immature DCs may induce anergy rather than an antitumor immune response, via secretion of Th2-type cytokines, whereas mature DCs, through high levels of MHC and costimulatory molecules, induce and sustain a specific immune response [29]. Studies that have used TNF- $\alpha$-treated DCs, which result in a mature $\mathrm{CD} 83^{+} \mathrm{DC}$ population [26], have reported encouraging clinical responses that were lacking from previous studies using immature DCs [22].

The optimal antigen(s) for incorporation into a DC vaccine for HCC is also an unresolved question. Expression of a number of antigens, including Mage-1 and -3, and NY-ESO1, has been demonstrated in HCC, but no single antigen is omnipresent and detailed knowledge of specific T-cell reactivity against many of the antigens is limited [30,31]. AFP is also a potential candidate antigen. One might expect that because AFP is a nonmutated self-antigen highly expressed in fetal liver, responsive $\mathrm{T}$ cells would be largely deleted. However, studies have reported that AFP-specific T cells can be detected in healthy individuals and in cirrhotic and HCC patients, and that these can be re-stimulated and expanded in patients with HCC even in the presence of high serum levels [22,32-34]. Studies have also demonstrated the ability to induce T-cell responses against AFP using AFP peptide-pulsed DCs [22]. The immunodominant epitopes from AFP are not well characterized. Published studies suggest that both dominant and subdominant epitopes are recognized by the human T-cell repertoire in patients with HCC resulting in expansion of IFN- $\gamma$-producing effector cells. The response is complex because $T$ cells specific for subdominant epitopes are of similar or higher avidity than those specific for immunodominant ones, and in vitro DCs stimulate broad responses [16]. Further, use of specific peptides may limit the applicability of a vaccine to patients with the appropriate specific HLA type.

Because no single antigen is ubiquitously expressed by HCC, some studies have used whole cell lysate as a source of multiple antigens (including AFP). An advantage of this approach is that DCs pulsed with whole protein will be more effective at eliciting antigenspecific responses from both CD 4 helper T cells and $\mathrm{CD} 8^{+}$cytotoxic $\mathrm{T}$ cells to multiple antigens, independent of HLA type. Activation of both $\mathrm{CD}^{+}$and $\mathrm{CD}^{+}{ }^{+} \mathrm{T}$ cells appears to be a prerequisite for a robust antitumor immune response 
[35] and the generation of $\mathrm{T}$ cells against multiple antigens may reduce the risk of tumor escape through downregulation of a single specific antigen. Indeed, a study of naturally occurring CD8 T-cell responses in a series of patients with HCC suggested that responses to a broader range of tumor-associated antigens may be positively associated with survival [36]. Several studies have used the hepatoblastoma cell line, HepG2. It expresses high levels of AFP and is readily available, making it a pragmatic choice for clinical studies. Studies have also shown that specific anti-HCC T cells can be generated in vitro by DCs pulsed with HepG2 total RNA [37]. Other liver cancer cell lines may equally be used. Autologous tumor lysate may be used but may be unpredictable in its availability and there is potential variability in antigen expression between individuals that might limit the investigation of T-cell responses. The use of whole cell lysate is associated with encouraging evidence of clinical responses whereas studies using HLA-restricted peptides failed to induce clinical responses, which may be a consequence of the lack of CD4 help. Conversely, whole cell lysate may increase the risk of autoimmunity through shared epitopes with normal tissues. However, this has not been observed in studies to date.

The optimal route of vaccination also remains to be determined. Because antigen presentation to $\mathrm{T}$ cells by DCs normally occurs in lymph nodes, it appears logical to deliver vaccine via the route that optimizes their access to regional nodes. However, it is not yet known whether this requires direct inoculation to the regional nodes (which for the liver may be technically challenging), or whether a more convenient route such as inguinal nodes, intradermal or intravascular (either through the hepatic artery or intravenously) might allow DCs to home to nodes. This process may be influenced by the maturation status of the DCs. Although priming in regional hepatic nodes may, in principle, generate T cells with preferential liver homing properties, $\mathrm{T}$ cells may also be primed by DC in the spleen and it is also not yet known whether priming in hepatic lymph nodes is more effective than in other sites.

Studies have shown that immature DCs injected into the tumor artery survive for several weeks within the tumor and are capable of generating T-cell responses against tumor antigens $[6,24,38]$. A study inoculating lysate-loaded DCs either intratumorally, subcutaneously or intravenously in a murine subcutaneous HCC model suggested intratumoral to be the optimal route. However, these DC were also engineered to express CD40 ligand, which may directly induce apoptosis and upregulate antigen-processing machinery in CD40-positive HCC cells when administered intratumorally and might explain the apparent superiority of this route [39-41]. Other studies suggest that DCs may be trapped inside the tumor and are unable to migrate to draining lymph nodes; thus, whether intratumoral therapy is more effective than systemic delivery is yet to be determined. Tracking studies to define the distribution of DCs administered via different routes are underway.

\section{- Dendritic cells with cytokine-induced killer cells}

Although DC-based vaccination is feasible, to date, clinical efficacy has been modest at best. Cytokine-induced killer cells (CIK) have the ability to kill autologous tumor cells in an MHC-independent manner, which may be important since downregulation of MHC may commonly contribute to low tumor immunogenicity. In vitro studies have confirmed that CIK derived from patients with AFP-secreting tumors can be primed by AFP-loaded DC to recognize and lyse an AFP-expressing HCC cell line suggesting this to be a potential approach to enhance antitumor immune responses [42]. This approach has been tested in a small clinical trial of nine patients who received immunotherapy following standard chemo- or radio-therapy. The immunotherapy approach was complex, comprising bone marrow-derived CIK activated ex vivo by co-culture with DC derived from PBMC loaded with autologous tumor cell lysate from surgically removed tumor specimens. Treatment was safe and well-tolerated, tumorspecific immune responses could be elicited and an impact on survival compared with nine 'control' patients who did not receive immunotherapy was claimed (median survival 17.1 vs 10.1 months), although treatment was not randomly assigned and patient numbers were too small to allow meaningful comparison [43].

\section{- NK cells}

The direct application of NK cell-based immunotherapy for HCC is also being investigated. NK cells may potentially recognize and kill tumor cells through a number of mechanisms: absence of MHC class I on tumor 
cells (the 'missing self' hypothesis); expression of ligands for appropriate activating receptors expressed by NK cells; expression of 'danger signals' such as Toll-like receptors within the tumor microenvironment; or through sensitivity of tumor cells to TRAIL-induced apoptosis. Studies suggest that allogeneic liver-derived NK cells activated ex vivo by IL-2 may be more potent than autologous liver- or peripheral blood-derived NK cells and clinical studies suggest that isolation of such cells from donor liver perfusate prior to liver transplantation is feasible and may be worthy of study as adjuvant immunotherapy for patients with HCC undergoing transplantation [44]. Other studies suggest that Toll-like receptor, TLR-3 activation may positively modulate endogenous NK cells and the effect of TLR-3 ligation may be worthy of further study [45].

\section{- Genetically modified T cells}

Genetic modification of peripheral blood T cells to express antigen-specific T-cell receptors has demonstrated clinical responses in pilot studies for patients with malignant melanoma, although the approach is currently labor-intensive, expensive and confined to specialist centers. Similarly, in preclinical studies, $\mathrm{T}$ cells have been engineered to express TCR targeting hepatitis B virus envelope antigen as a potential immunotherapeutic approach for patients with $\mathrm{HBV}$ associated HCC. However, since expression of viral antigens is not confined to malignant hepatocytes, there are concerns of hepatitis from such an approach and T-cell therapy against tumorspecific antigens may be a preferred approach for further clinical development, though is currently restricted by a limited choice of suitable target antigens and, indeed, for such an approach to be successful $T$ cells redirected against a number of antigens may be required [46].

Non-cell-based immunotherapy

Several other strategies aiming to stimulate the immune response against cancer do not use autologous cells as the therapeutic product (Figure 2).

\section{- Cytokines}

Cytokines are secreted proteins used by immune system cells for intercellular communication. A number of them have potent immunostimulatory and antineoplastic activities in animal models and the results of several Phase I and II clinical trials in patients with gastrointestinal cancer have been reported and recently reviewed [47]. IFN- $\alpha$ was initially considered a promising treatment for cancer - and specifically for HCC - due to its antiproliferative, proapoptotic and immunomodulatory activities. However, it was not effective in reducing postoperative recurrence of viral hepatitis-related HCC [48] and had no effect on tumor progression rate and survival among patients with inoperable advanced HCC [49] at intermediate doses. A beneficial effect might be expected at a higher dose [50], but tolerance is excessively poor. Furthermore, the combination of IFN- $\alpha$ with cisplatin, fluorouracil and doxorubicin was no better than doxorubicin alone as first-line treatment of unresectable HCC patients [51]. Other cytokines have not been evaluated specifically in HCC but produced no signal of beneficial effects in other gastrointestinal tumors. This includes regimens incorporating IFN- $\beta$ and IFN- $\gamma$, TNF- $\alpha$, GM-CSF and IL-2.

\section{- Oncolytic viruses}

Oncolytic viruses are naturally found or much more frequently genetically modified viruses that replicate with some degree of selectivity in malignant cells and induce their killing. Cytokines can be integrated as transgenes in oncolytic viruses to enhance the intrinsic immunostimulatory effect of active viral replication and the detection of its pathogen-associated molecular patterns. Viral replication occurs after intratumoral or even intra-arterial injection of these agents [52]. Oncolytic viruses have been developed from adenovirus, herpesvirus, vaccinia and others. Oncolytic poxviruses have progressed further than other antitumor agents in the clinical development pathway. They can replicate selectively in cancer cells and produce tumor cell necrosis and viral spread to tumor tissues [53]. Pexa-Vec (JX-594) is an oncolytic poxvirus engineered from the strain used for smallpox vaccination. It carries a disruption of the thymidine kinase gene that renders the virus partially dependent on cellular thymidine kinase, which is more abundant in cancer cells; and the human GM-CSF gene under the control of a synthetic promoter. The result is that Pexa-Vec may cause direct oncolysis and stimulate antitumoral immunity as a result of local GM-CSF expression. It attains tumor responses and enhances survival after intravenous and intratumoral administration in animal models [54]. In a Phase I clinical trial where 
Cytokines

IFN- $\alpha$
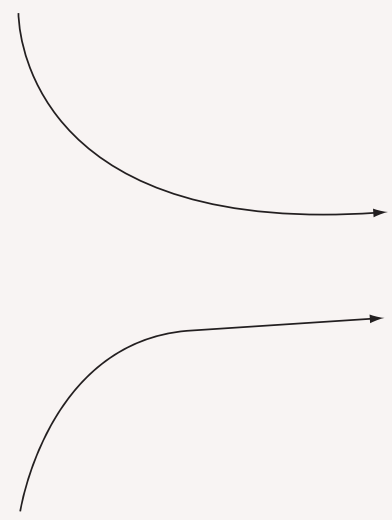

'Armed' oncolytic virus

Pexavec (GM-CSF-expressing vaccinia)
Peptide vaccination

Glypican 3

Multipeptide mixture

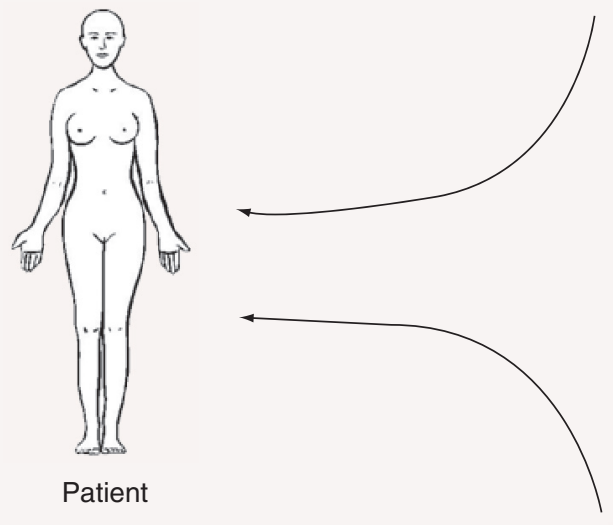

Checkpoint inhibition mAbs

Tremelimumab (anti-CTLA4)

Nivolumab (anti-PD1)

Figure 2. A summary of strategies used in non-cell-based immunotherapy of hepatocellular carcinoma.

patients with refractory primary or metastatic liver tumors were treated (including hepatocellular, colorectal, melanoma and lung carcinomas), objective tumor responses were shown in both injected and noninjected tumors [55] providing the proof of concept of the systemic potential of this approach. In a more detailed analysis of three patients with HCC and hepatitis B virus infection, one of them achieved an objective remission and the remaining two had a biological (FDG-PET) response [56]. Acute induction of circulating IL- $4,-6,-10$, TNF- $\alpha$ and IFN- $\gamma$ was noted within the first $1-3 \mathrm{~h}$ after injection. Unfortunately, subsequent results have not been so encouraging. The TRAVERSE study [57] is a Phase IIb clinical trial in which patients with advanced HCC who had failed sorafenib treatment were randomized to receive Pexa-Vec plus best supportive care or best supportive care alone. Although the results are not fully available, a press release acknowledged that the study did not meet its primary endpoint of a more prolonged overall survival.

\section{- Peptide vaccines}

$\mathrm{CD}^{+} \mathrm{T}$ cells may recognize tumor-associated antigens and kill tumor cells. Tumor-associated antigens are self-derived proteins rendered immunogenic in tumors by mutation or aberrant expression [58]. In HCC patients, several tumorassociated antigens can spontaneously induce $\mathrm{CD}^{+}$T-cell responses including AFP, glypican-3 (GPC-3), melanoma-associated geneA1 (MAGE-A1) [59-61]. Some of these tumorassociated antigens have been used for peptide vaccination.

Glypican-3 (GPC3) belongs to the family of heparan sulfate proteoglycans present in the surface of hepatocytes and other cells. Interestingly enough GPC3 is overexpressed in up to $80 \%$ of HCC tumors and indicates a worse prognosis [62]. This overexpression may help differentiate HCC from nonmalignant dysplastic nodules [63] and GPC3 immunohistochemistry is increasingly used for diagnostic purposes. Claimed as potentially valuable serum tumor marker, it has not been validated for screening purposes. From a therapeutic perspective, both a vaccine and a monoclonal antibody approach are under development. Two GPC3-derived epitopes that bind to HLA-A2 and HLA-A24 [64] and are able to induce antibody-dependent cellular cytotoxicity [65] were used in a pilot therapeutic vaccination clinical trial in which one or the other was used depending on patient's HLA haplotype [66]. The safety profile was not surprisingly excellent with only $12 \%$ of patients experiencing grade 3 adverse events (increased transaminases 
or bilirubin). Promisingly, an objective partial response was observed among 33 treated patients and four additional patients showed signs of tumor response or necrosis that did not qualify for a partial response. Furthermore, GPC3specific T-cell responses were observed in 91\% of patients and GPC3-specific CTL frequency correlated with survival. Following these observations, an ongoing Phase II clinical trial is allegedly exploring the efficacy as an adjuvant therapy after resection.

Single or multiple peptides derived from tumor-associated antigens may be used for cancer vaccination, generally in combination with adjuvants like Freund's or poly(I:C). One such vaccine consisting of multiple tumor-associated peptides naturally presented in human renal cell cancer tissue has been tested in a randomized Phase II trial that confirmed that immune responses to multiple tumor-associated peptides were associated with longer overall survival [67]. This approach is now being explored in HCC patients in an ongoing EU Project (HEPAVAC) [68].

\section{- T-cell checkpoint inhibitors}

Monoclonal antibodies that act by blocking the negative signals of T-cell checkpoints probably draw the highest expectations in cancer immunotherapy nowadays [69]. Targeting cytotoxic T-lymphocyte-associated antigen 4 (CTLA-4), programmed death 1 (PD-1) and programmed death ligand 1 (PD-L1) by use of monoclonal antibodies has revolutionized the field of cancer immunotherapy in the last few years. Tremelimumab and ipilimumab are human monoclonal antibodies that bind to CTLA-4. At the immune synapses formed by the T lymphocyte and the antigen-presenting cells, the role of CTLA- 4 is to outcompete the binding of the CD28 costimulatory receptor to CD80 and CD 86 [70]. In addition, binding of CTLA-4 and $\mathrm{B} 7$ sends an inhibitory signal that serves as a natural brake for T-cell activation [71]. CTLA-4 blockade by monoclonal antibodies releases this brake and enhances T-cell activation and proliferation through various mechanisms still under study [72,73], which may include partial intratumoral depletion of regulatory $\mathrm{T}$ cells [74].

Tremelimumab was shown to produce tumor responses among patients with metastatic melanoma [75] and metastatic colorectal cancer [76] while ipilimumab (another CTLA-4blocking $\mathrm{mAb}$ ) demonstrated a survival advantage over a gp100 vaccine among patients with advanced melanoma [77]. In a pilot Phase II clinical trial targeting the population of HCC patients with chronic hepatitis $\mathrm{C}$ virus infection, tremelimumab has recently shown signs of antitumor and antiviral activity [78]. Twenty-one patients with mostly advanced tumors ( $57 \%$ were on BCLC stage C) and varying degrees of liver dysfunction ( $43 \%$ were on Child-Pugh class B) received tremelimumab at a dose of $15 \mathrm{mg} / \mathrm{kg}$ every 90 days until tumor progression or unacceptable toxicity. Treatment was overall well tolerated with an itching skin rash being the most frequent adverse event. Transaminases peaked transiently after the first dose in more than half the patients (grade 3 or higher in $45 \%$ of cases) but did not lead to liver failure or recurred in following cycles. The reason for this liver insult is obscure since it was not associated with an increase in viral load or with significant changes in circulating cytokines, leaving an overproduction of proinflammatory cytokines by intrahepatic immune cells the most likely explanation.

Despite the reduced number of patients, revealing signs of efficacy were observed. Three patients (18\%) had a partial response that was durable in two cases (9.2 and 15.8 months) and 10 patients $(59 \%)$ had a stable disease that in almost half of the cases lasted longer than 6 months. Furthermore, a $>50 \%$ drop in $\alpha$-fetoprotein was observed in $36 \%$ of patients with high baseline levels (>100 ng/ml). The median time to progression of 6.5 months (95\% CI: 3.95-9.14) compares well with most Phase II trials of targeted agents and provides further evidence of antitumor activity. Tumor-specific responses were not tested but specific T-cell responses against hepatitis $\mathrm{C}$ virus antigens were observed in most patients. Those patients with absent or minor reductions in circulating IFN- $\gamma$ post-treatment showed a significantly better tumor response than those with a higher than $50 \%$ reduction.

\section{- Other monoclonal antibodies}

Monoclonal antibodies may be used to block inhibitory T-cell checkpoints other than CTLA-4 including PD-1 and PD-L1. PD-1 receptor is expressed by $\mathrm{T}$ lymphocytes preferentially with long-term exposure to antigens and contributes to T-cell exhaustion. Its primary ligand PD-L1 is commonly expressed in the tumor microenvironment, including 
cancer cells and tumor-infiltrating macrophages. Antibodies that block the interaction between PD-1 and PD-L1 in tumors have shown substantial clinical antitumor activity [79]. Nivolumab and lambrolizumab, respectively a fully human and a humanized antibody that block PD-1, have produced impressive durable objective responses in patients with melanoma $[80,81]$, renal-cell cancer and non-small-cell lung cancer [81]. Following these results, a dose-escalating Phase II clinical trial is exploring the safety and efficacy of nivolumab for the treatment of HCC, including cohorts that explore viral etiology cases [82].

GC33 is a human monoclonal antibody that binds GPC3 and has been tested in a small pilot Phase I trial involving 20 patients with advanced stage HCC [83]. Patients received GC33 in escalating doses of $2.5-20 \mathrm{mg} / \mathrm{kg}$ given weekly. No relevant or dose-limiting toxicities were observed. Although no objective tumor responses were observed, time to progression was 26 weeks among patients with high GPC3 staining and only 7.1 weeks among those with low GPC3 staining, suggesting that a delayed tumor growth could have been induced by the treatment. Further clinical development is now awaited. An international Phase II clinical trial exploring whether GC33 as a second-line systemic treatment [84] may increase progression-free survival among patients with advanced tumors is underway. As it is a Phase I dose-finding clinical trial exploring the combination of GC33 and sorafenib [85].

HN3 is another monoclonal antibody that has been shown to prevent proliferation of GPC3-positive tumor cells but not of tumor cells that lack GPC3 or cells that had their GPC3 expression knocked down. This nonimmune mechanism of action is based in cell-cycle arrest through inactivation of yap, a Hippo pathway member [86].

\section{Conclusion \& future perspective}

There are currently four main areas of progress in cancer immunotherapy: vaccination with individual tumor antigens; more immunogenic adjuvants for vaccine formulation; immunomodulation with immunostimulatory monoclonal antibodies; improved cell-based therapies; and combinatorial approaches of immunotherapy.

Individual or private tumor antigens are the product of genetic alterations occurring in the tumor cells of an individual patient rather than the conventionally identified shared differentiation or embryonic/fetal proteins. Such private antigens may be the result of tumorspecific post-translational modifications but are much more often the result of mutations. Full exome sequencing shows that the genome of human solid tumors usually harbors over 100 such mutations expressed at the protein level. A number of those mutations give rise to peptides that can be presented by the patient's HLA allele products to specific $T$ lymphocytes. Technology has reached the point in which individual tumor antigens can be affordably identified by sequencing exons or eluting the HLA-bound peptides and sequencing them by MS [87]. Bioinformatics play a crucial role for epitope prediction. To make the most of these techniques both HLA class I and II epitopes are to be identified to GMP synthetize polypeptides containing them [87]. Longer peptides than the minimally restricted epitope are preferred based on experience [88]. HCC is no exception and individual (private) tumor antigens are to be identified in each patient and the approach should be tested.

The most appealing approach is probably the identification of the HLA-ligandome [89] that includes the identification of naturally presented HLA-associated peptides from primary cancer cells and the subsequent selection of tumorassociated peptides by differential gene expression analysis and data mining followed by validation of selected candidates through monitoring in vivo $\mathrm{T}$-cell responses in the context of patientindividualized immunizations. The final aim is to develop universal or personalized vaccines comprising multiple tumor-associated epitopes in order to induce a broad and specific immune response against cancer. This approach has already resulted in therapeutic vaccines for renal cell cancer able to induce immune responses that were associated with a more prolonged survival [67]. A randomized Phase III study is underway in renal cell cancer patients.

On the other hand, adjuvants for cancer vaccines are elements that enhance the ability of a given antigen to induce an immune response mediated by lymphocytes producing IFN- $\gamma$ and capable of mediating cytolysis. The most powerful approaches involve mimicry of viral and intracellular bacterial infection [90] and antigen delivery to the proper professional antigen-presenting cells [91]. Pathogen danger signals can be imitated by adding into the vaccine nucleic acids with microbial characteristics and other biomolecules sensed as alien by innate 
proinflammatory receptors [92]. Receptors for pathogen patterns turn on the convenient form of local inflammation that results in sufficiently intense cellular immunity toward the antigens present in the vaccine inoculum. Two new formulations are gaining momentum in clinical trials: recombinant viruses that frequently encode both antigens and immunostimulatory cytokines [93] and synthetic mRNA encoding tumor antigens [94]. Another emerging important topic is routing vaccines so to procure the traffic of activated lymphocytes to the tissue where the malignancy originates or nests [95].

Immunomodulatory antibodies come in two flavors, namely, antagonists for co-inhibitory receptors or check-points [72] and agonists for co-stimulatory molecules [96,97]. These agents are under intensive clinical trial development. For HCC, a single trial with a check-point inhibitor directed to CTLA-4 (tremelimumab) has been reported. Signs of clinical activity and a favorable safety profile were observed even after having used a probably suboptimal regimen. A clinical trial testing the PD-1 mAb nivolumab is ongoing [98] and its results are eagerly awaited. Other co-inhibitory (check-point) targets for immunostimulatory $\mathrm{mAb}$ are entering the clinic although none of them have been tried for HCC so far. TIM-3 is a surface glycoprotein overexpressed in exhausted tumor infiltrating $\mathrm{T}$ lymphocytes at least in melanoma whose signaling contributes to the dysfunctional phenotype [99]. Galectin-9 binding to TIM-3 provides negative signals and if this interaction if disrupted with monoclonal antibodies antitumor effects have been reported in mice [100], in particular when combined with PD-1 blockade. LAG-3 is a protein expressed by activated $\mathrm{T}$ lymphocytes that binds MHC class II molecules with high affinity. Disruption of LAG-3 ligation with monoclonal antibodies restores T-cell functions and successfully treats transplanted models of cancer [101], again more evidently so when an antiPD-1 monoclonal antibody is combined [102]. OX40, on the contrary, is a surface protein of the TNF-receptor family expressed on activated $\mathrm{T}$ cells involved in providing co-stimulation. Its ligation with agonist monoclonal antibodies or its natural ligand gives rise to enhanced antitumor immunity in mouse models [103]. Recently, results in a clinical trial with single doses of a xenogenic mouse monoclonal antibody suggests augmentation of antitumor immunity in several types of cancer that do not include HCC [104].
The field of agonist immunostimulatory monoclonal antibodies for HCC remains completely unexplored and surely will include in the future mAbs directed to CD40, CD137, OX40 and glucocorticoid-induced TNF receptor familyrelated gene (GITR) [96,97].

On the other hand, cellular therapies provide a practical basis for a therapeutic tumor vaccine [105]. There is evidence of safety and immune activity, with some evidence of clinical response for patients with HCC. Immune activity and clinical responses in a proportion of patients with advanced tumors and chronic liver disease are encouraging and suggest that future trials in less advanced disease may be associated with better clinical responses. In the future, immunotherapy should probably be used as an adjuvant to radical therapy. In part because the reduced tumor burden is likely to be more responsive to immunotherapy but also due to the fact that local ablation causes the release of tumor antigens that evoke antitumor immune responses [106-108]. Intra-arterial injection of matured DC during transarterial embolization in patients with hepatitis $\mathrm{C}$ virus-related HCC was followed by increased survival in a small group of patients compared with historical controls [24].

Immunotherapy as an adjuvant to surgical resection may require greater caution, because liver autoimmunity has been reported in response to AFP DNA vaccination in a mouse model of liver regeneration following partial hepatectomy, a setting known to be associated with increased AFP expression [109], although other studies suggest no impairment of liver regeneration in this context [110]. Further improvements in efficacy may come from combinatorial approaches to generating antitumor immune responses in which DC vaccination is combined with strategies to overcome regulatory $\mathrm{T}$ cells or the inhibitory effect of Th2 cytokines, or with conditioning chemotherapy [18] or in combination with T-cell checkpoint blockade.

There is consensus that combination immunotherapy strategies are to be developed [111]. A combination of CTLA-4 and PD-1 blockade is very active against malignant melanoma with manageable safety [112]. Such a spectacular Phase I trial has amplified our excitement on this sort of immunotherapy combinations. Preclinical research in rodents is full of examples of treatment in which synergy can be attained. Combinations of immunostimulatory $\mathrm{mAb}$ in conjunction with vaccines, 
adoptive T-cell therapy or conventional therapies are feasible and need to be tested. Recent evidence in mice developing spontaneous HCC has made the proof of concept for curative synergistic combinations of an immunostimulatory mAb triplet combination (PD-L1 + CD137 + OX40) in conjunction with adoptive T-cell therapy [113]. Safety and cost hurdles are to be considered but there is no doubt that combinatorial immunotherapy approaches have an enormous potential for HCC as they have for other solid tumors.
Financial \& competing interests disclosure CIBERehd is funded by Instituto de Salud Carlos III. Bruno Sangro has received consulting fees from Medimmune and Bristol-Myers-Squibb. Ignacio Melero has received consulting fees from Bristol-Myers-Squibb, Astra Zeneca, Genentech and Roche. The authors have no other relevant affiliations or financial involvement with any organization or entity with a financial interest in or financial conflict with the subject matter or materials discussed in the manuscript apart from those disclosed.

No writing assistance was utilized in the production of this manuscript.

\section{References}

1 Dunn GP, Bruce AT, Ikeda H, Old LJ, Schreiber RD. Cancer immunoediting: from immunosurveillance to tumor escape. Nat. Immunol. 3(11), 991-998 (2002).

2 Buonaguro L, Petrizzo A, Tornesello ML, Buonaguro FM. Translating tumor antigens into cancer vaccines. Clin. Vaccine Immunol. 18(1), 23-34 (2011).

3 Yoong KF, Afford SC, Jones R et al. Expression and function of CXC and CC chemokines in human malignant liver tumors: a role for human monokine induced by gamma-interferon in lymphocyte recruitment to hepatocellular carcinoma. Hepatology 30 (1), 100-111 (1999).

4 Wada Y, Nakashima O, Kutami R, Yamamoto O, Kojiro M. Clinicopathological study on hepatocellular carcinoma with lymphocytic infiltration. Hepatology 27(2), 407-414 (1998).

5 Friedl J, Stift A, Paolini P et al. Tumor antigen pulsed dendritic cells enhance the cytolytic activity of tumor infiltrating lymphocytes in human hepatocellular cancer. Cancer Biother. Radiopharm. 15(5), 477-486 (2000).

6 Feijoó E, Alfaro C, Mazzolini G et al. Dendritic cells delivered inside human carcinomas are sequestered by interleukin- 8 . Int. J. Cancer 116(2), 275-281 (2005).

7 Strand S, Hofmann WJ, Hug $\mathrm{H}$ et al. Lymphocyte apoptosis induced by CD95 (APO-1/Fas) ligand-expressing tumor cells - a mechanism of immune evasion? Nat. Med. 2(12), 1361-1366 (1996).

8 Shiraki K, Yamanaka T, Inoue $\mathrm{H}$ et al. Expression of TNF-related apoptosis-inducing ligand in human hepatocellular carcinoma. Int. J. Oncol. 26(5), 1273-1281 (2005).

9 Wang B-J, Bao J-J, Wang J-Z et al. Immunostaining of PD-1/PD-Ls in liver tissues of patients with hepatitis and hepatocellular carcinoma. World J. Gastroenterol. 17(28), 3322-3329 (2011).

10 Gao Q, Wang X-Y, Qiu S-J et al. Overexpression of PD-L1 significantly associates with tumor aggressiveness and postoperative recurrence in human hepatocellular carcinoma. Clin. Cancer Res. 15(3), 971-979 (2009).

11 Zhou J, Ding T, Pan W, Zhu L-Y, Li L, Zheng L. Increased intratumoral regulatory $\mathrm{T}$ cells are related to intratumoral macrophages and poor prognosis in hepatocellular carcinoma patients. Int. J. Cancer 125(7), 1640-1648 (2009).

12 Pedroza-Gonzalez A, Verhoef C, Ijzermans JNM et al. Activated tumor-infiltrating CD4+ regulatory $\mathrm{T}$ cells restrain antitumor immunity in patients with primary or metastatic liver cancer. Hepatology 57(1), 183-194 (2013).

13 Zhao W, Zhang L, Xu Y et al. Hepatic stellate cells promote tumor progression by enhancement of immunosuppressive cells in an orthotopic liver tumor mouse model. Lab. Invest. 94(2), 182-191 (2014).

14 Cariani E, Pilli M, Zerbini A et al. Immunological and molecular correlates of disease recurrence after liver resection for hepatocellular carcinoma. PLoS ONE 7(3), e32493 (2012).

15 Chen Y-X, Man K, Ling GS et al. A crucial role for dendritic cell (DC) IL-10 in inhibiting successful DC-based immunotherapy: superior antitumor immunity against hepatocellular carcinoma evoked by DC devoid of IL-10. J. Immunol. 179(9), 6009-6015 (2007).

16 Liu Y, Daley S, Evdokimova VN, Zdobinski DD, Potter DM, Butterfield LH. Hierarchy of alpha fetoprotein (AFP)-specific T cell responses in subjects with AFP-positive hepatocellular cancer. J. Immunol. 177(1), 712-721 (2006).
17 Han Y, Chen Z, Yang Y et al. Human CD14+ CTLA-4+ regulatory dendritic cells suppress T-cell response by cytotoxic T-lymphocyte antigen-4-dependent IL-10 and indoleamine2,3-dioxygenase production in hepatocellular carcinoma. Hepatology 59(2), 567-579 (2014).

18 Cerundolo V, Hermans IF, Salio M. Dendritic cells: a journey from laboratory to clinic. Nat. Immunol. 5(1), 7-10 (2004).

19 Nestle FO, Alijagic S, Gilliet M et al. Vaccination of melanoma patients with peptideor tumor lysate-pulsed dendritic cells. Nat. Med. 4(3), 328-332 (1998).

20 Small EJ, Fratesi P, Reese DM et al. Immunotherapy of hormone-refractory prostate cancer with antigen-loaded dendritic cells. J. Clin. Oncol. 18(23), 3894-3903 (2000).

21 Höltl L, Rieser C, Papesh C et al. Cellular and humoral immune responses in patients with metastatic renal cell carcinoma after vaccination with antigen pulsed dendritic cells. J. Urol. 161(3), 777-782 (1999).

22 Butterfield LH, Ribas A, Dissette VB et al. A Phase I/II trial testing immunization of hepatocellular carcinoma patients with dendritic cells pulsed with four alphafetoprotein peptides. Clin. Cancer Res. 12(9), 2817-2825 (2006).

23 Lee W-C, Wang H-C, Hung C-F, Huang P-F, Lia C-R, Chen M-F. Vaccination of advanced hepatocellular carcinoma patients with tumor lysate-pulsed dendritic cells: a clinical trial. J. Immunother. 28(5), 496-504 (2005).

24 Nakamoto Y, Mizukoshi E, Tsuji H et al. Combined therapy of transcatheter hepatic arterial embolization with intratumoral dendritic cell infusion for hepatocellular carcinoma: clinical safety. Clin. Exp. Immunol. 147(2), 296-305 (2007).

25 Kantoff PW, Higano CS, Shore ND et al. Sipuleucel-T immunotherapy for castrationresistant prostate cancer. N. Engl. J. Med. 363(5), 411-422 (2010). 
26 Palmer DH, Midgley RS, Mirza N et al. A Phase II study of adoptive immunotherapy using dendritic cells pulsed with tumor lysate in patients with hepatocellular carcinoma. Hepatology 49(1), 124-132 (2008).

27 Ansary El M, Mogawer S, Elhamid SA et al. Immunotherapy by autologous dendritic cell vaccine in patients with advanced HCC. J. Cancer Res. Clin. Oncol. 139(1), 39-48 (2013).

28 Bray SM, Vujanovic L, Butterfield LH. Dendritic cell-based vaccines positively impact natural killer and regulatory T cells in hepatocellular carcinoma patients. Clin. Dev. Immunol. 2011, 249281 (2011).

29 Dhodapkar MV, Steinman RM. Antigenbearing immature dendritic cells induce peptide-specific CD8(+) regulatory T cells in vivo in humans. Blood $100(1), 174-177$ (2002).

30 Luo G, Huang S, Xie X et al. Expression of cancer-testis genes in human hepatocellular carcinomas. Cancer Immun. 2, 11 (2002).

31 Evdokimova VN, Liu Y, Potter DM, Butterfield LH. AFP-specific CD 4 + helper T-cell responses in healthy donors and HCC patients. J. Immunother. 30 (4), 425-437 (2007).

32 Hanke P, Rabe C, Serwe M et al. Cirrhotic patients with or without hepatocellular carcinoma harbour AFP-specific T-lymphocytes that can be activated in vitro by human alpha-fetoprotein. Scand. J. Gastroenterol. 37(8), 949-955 (2002).

33 Butterfield LH, Ribas A, Meng WS et al. T-cell responses to HLA-A*0201 immunodominant peptides derived from alpha-fetoprotein in patients with hepatocellular cancer. Clin. Cancer Res. 9(16 Pt 1), 5902-5908 (2003).

34 Iwashita Y, Tahara K, Goto S et al. A Phase I study of autologous dendritic cell-based immunotherapy for patients with unresectable primary liver cancer. Cancer Immunol. Immunother. 52(3), 155-161 (2003).

35 Schumacher L, Ribas A, Dissette VB et al. Human dendritic cell maturation by adenovirus transduction enhances tumor antigen-specific T-cell responses. J. Immunother. 27(3), 191-200 (2004).

36 Flecken T, Schmidt N, Hild S et al. Immunodominance and functional alterations of tumor-associated antigenspecific CD8 + T-cell responses in hepatocellular carcinoma. Hepatology 59(4), 1415-1426 (2014).

37 Zhang L, Zhang H, Liu W et al. Specific antihepatocellular carcinoma $\mathrm{T}$ cells generated by dendritic cells pulsed with hepatocellular carcinoma cell line HepG2 total RNA. Cell. Immunol. 238(1), 61-66 (2005).

38 Chi K-H, Liu S-J, Li C-P et al. Combination of conformal radiotherapy and intratumoral injection of adoptive dendritic cell immunotherapy in refractory hepatoma. J. Immunother. 28(2), 129-135 (2005).

39 Gonzalez-Carmona MA, Lukacs-Kornek V, Timmerman A et al. CD40ligand-expressing dendritic cells induce regression of hepatocellular carcinoma by activating innate and acquired immunity in vivo. Hepatology 48(1), 157-168 (2008).

40 Elmetwali T, Young LS, Palmer DH. CD40 ligand-induced carcinoma cell death: a balance between activation of TNFRassociated factor (TRAF) 3-dependent death signals and suppression of TRAF6-dependent survival signals. J. Immunol. 184(2), 1111-1120 (2010).

41 Hill SC, Youde SJ, Man S et al. Activation of CD40 in cervical carcinoma cells facilitates CTL responses and augments chemotherapyinduced apoptosis. J. Immunol. 174(1), 41-50 (2005).

42 Gonzalez-Carmona MA, Märten A, Hoffmann P et al. Patient-derived dendritic cells transduced with an a-fetoproteinencoding adenovirus and co-cultured with autologous cytokine-induced lymphocytes induce a specific and strong immune response against hepatocellular carcinoma cells. Liver Int. 26(3), 369-379 (2006).

43 Qiu Y, Xu M-B, Yun MM et al. Hepatocellular carcinoma-specific immunotherapy with synthesized $\alpha 1,3$ galactosyl epitope-pulsed dendritic cells and cytokine-induced killer cells. World J. Gastroenterol. 17(48), 5260-5266 (2011).

44 Ohira M, Nishida S, Tryphonopoulos P et al. Clinical-scale isolation of interleukin-2stimulated liver natural killer cells for treatment of liver transplantation with hepatocellular carcinoma. Cell Transplant. 21(7), 1397-1406 (2012).

45 Chew V, Tow C, Huang C et al. Toll-like receptor 3 expressing tumor parenchyma and infiltrating natural killer cells in hepatocellular carcinoma patients. J. Natl Cancer Inst. 104(23), 1796-1807 (2012).

46 Koh S, Shimasaki N, Suwanarusk R et al. A practical approach to immunotherapy of hepatocellular carcinoma using $\mathrm{T}$ cells redirected against hepatitis B virus. Mol. Ther. Nucleic Acids 2, e114 (2013).

47 Hernández-Alcoceba R, Sangro B, Berraondo P, Gonzalez-Aseguinolaza G, Prieto
J. Cytokines for the treatment of gastrointestinal cancers: clinical experience and new perspectives. Expert Opin. Investig. Drugs 22(7), 827-841 (2013).

48 Chen L-T, Chen M-F, Li L-A et al. Long-term results of a randomized, observationcontrolled, Phase III trial of adjuvant interferon alfa- $2 \mathrm{~b}$ in hepatocellular carcinoma after curative resection. Ann. Surg. 255(1), 8-17 (2012).

49 Llovet JM, Sala M, Castells L et al. Randomized controlled trial of interferon treatment for advanced hepatocellular carcinoma. Hepatology 31(1), 54-58 (2000).

50 Lai CL, Lau JY, Wu PC et al. Recombinant interferon-alpha in inoperable hepatocellular carcinoma: a randomized controlled trial. Hepatology 17(3), 389-394 (1993).

51 Yeo W, Mok TS, Zee B et al. A randomized Phase III study of doxorubicin versus cisplatin/interferon-2b/doxorubicin/ fluorouracil (PIAF) combination chemotherapy for unresectable hepatocellular carcinoma. J. Natl Cancer Inst. 97(20), 1532-1538 (2005).

52 Reid T, Galanis E, Abbruzzese J et al. Hepatic arterial infusion of a replicationselective oncolytic adenovirus (d11520): Phase II viral, immunologic, and clinical endpoints. Cancer Res. 62 (21), 6070-6079 (2002).

53 Kirn DH, Thorne SH. Targeted and armed oncolytic poxviruses: a novel multimechanistic therapeutic class for cancer. Nat. Rev. Cancer 9(1), 64-71 (2009).

54 Kim YI, Chung JW, Park JH, Han JK, Hong JW, Chung H. Intraarterial gene delivery in rabbit hepatic tumors: transfection with nonviral vector by using iodized oil emulsion. Radiology 240 (3), 771-777 (2006).

55 Park B-H, Hwang T, Liu T-C et al. Use of a targeted oncolytic poxvirus, JX-594, in patients with refractory primary or metastatic liver cancer: a Phase I trial. Lancet Oncol. 9(6), 533-542 (2008).

56 Liu T-C, Hwang T, Park B-H, Bell J, Kirn DH. The targeted oncolytic poxvirus JX-594 demonstrates antitumoral, antivascular, and anti-HBV activities in patients with hepatocellular carcinoma. Mol. Ther. 16(9), 1637-1642 (2008).

57 A Phase $2 b$ study of modified vaccinia virus to treat patients advanced liver cancer who failed sorafenib (TRAVERSE). http://clinicaltrials.gov/show/NCT01387555

58 Van den Eynde BJ, van der Bruggen P. T cell defined tumor antigens. Curr. Opin. Immunol. 9(5), 684-693 (1997). 
59 Thimme R, Neagu M, Boettler T et al. Comprehensive analysis of the alphafetoprotein-specific CD8 + T cell responses in patients with hepatocellular carcinoma. Hepatology 48(6), 1821-1833 (2008).

$60 \mathrm{Xu} \mathrm{Y,} \mathrm{Li} \mathrm{H,} \mathrm{Gao} \mathrm{RL,} \mathrm{Adeyemo} \mathrm{O,} \mathrm{Itkin} \mathrm{M,}$ Kaplan DE. Expansion of interferongamma-producing multifunctional $\mathrm{CD} 4+$ T-cells and dysfunctional CD $8+$ T-cells by glypican-3 peptide library in hepatocellular carcinoma patients. Clin. Immunol. 139(3), 302-313 (2011).

61 Zerbini A, Pilli M, Soliani P et al. Ex vivo characterization of tumor-derived melanoma antigen encoding gene-specific CD8+cells in patients with hepatocellular carcinoma. J. Hepatol. 40 (1), 102-109 (2004).

62 Capurro M, Wanless IR, Sherman M et al. Glypican-3: a novel serum and histochemical marker for hepatocellular carcinoma. Gastroenterology 125(1), 89-97 (2003).

63 International Consensus Group for Hepatocellular Neoplasia. Pathologic diagnosis of early hepatocellular carcinoma: A report of the international consensus group for hepatocellular neoplasia. Hepatology 49(2), 658-664 (2008).

64 Komori H, Nakatsura T, Senju S et al. Identification of HLA-A2- or HLA-A24restricted CTL epitopes possibly useful for glypican-3-specific immunotherapy of hepatocellular carcinoma. Clin. Cancer Res. 12(9), 2689-2697 (2006).

65 Nakano K, Orita T, Nezu J et al. Antiglypican 3 antibodies cause ADCC against human hepatocellular carcinoma cells. Biochem. Biophys. Res. Commun. 378(2), 279-284 (2009).

66 Sawada Y, Yoshikawa T, Nobuoka D et al. Phase I trial of a glypican-3-derived peptide vaccine for advanced hepatocellular carcinoma: immunologic evidence and potential for improving overall survival. Clin. Cancer Res. 18(13), 3686-3696 (2012).

67 Walter S, Weinschenk T, Stenzl A et al. Multipeptide immune response to cancer vaccine IMA901 after single-dose cyclophosphamide associates with longer patient survival. Nat. Med. 18(8), 1254-1261 (2012).

68 HEPAVAC. Cancer vaccine development for hepatocellular carcinoma. www.hepavac.eu

69 Melero I, Hervás-Stubbs S, Glennie M, Pardoll DM, Chen L. Immunostimulatory monoclonal antibodies for cancer therapy. Nat. Rev. Cancer 7(2), 95-106 (2007).
70 Chambers CA, Kuhns MS, Egen JG, Allison JP. CTLA-4-mediated inhibition in regulation of $\mathrm{T}$ cell responses: mechanisms and manipulation in tumor immunotherapy. Annu. Rev. Immunol. 19, 565-594 (2001).

71 Marengère LE, Waterhouse P, Duncan GS, Mittrücker HW, Feng GS, Mak TW. Regulation of $\mathrm{T}$ cell receptor signaling by tyrosine phosphatase SYP association with CTLA-4. Science 272(5265), 1170-1173 (1996).

72 Pardoll DM. The blockade of immune checkpoints in cancer immunotherapy. Nat. Rev. Cancer. 12(4), 252-264 (2012).

73 Walker LSK, Sansom DM. The emerging role of CTLA4 as a cell-extrinsic regulator of T cell responses. Nat. Rev. Immunol. 11(12), 852-863 (2011).

74 Quezada SA, Peggs KS, Simpson TR, Shen Y, Littman DR, Allison JP. Limited tumor infiltration by activated $\mathrm{T}$ effector cells restricts the therapeutic activity of regulatory T cell depletion against established melanoma. J. Exp. Med. 205(9), 2125-2138 (2008).

75 Camacho LH, Antonia S, Sosman J et al. Phase I/II trial of Tremelimumab in patients with metastatic melanoma. J. Clin. Oncol. 27(7), 1075-1081 (2009).

76 Chung KY, Gore I, Fong L et al. Phase II study of the anti-cytotoxic T-lymphocyteassociated antigen 4 monoclonal antibody, tremelimumab, in patients with refractory metastatic colorectal cancer. J. Clin. Oncol. 28(21), 3485-3490 (2010).

77 Hodi FS, O'Day SJ, McDermott DF et al. Improved survival with Ipilimumab in patients with metastatic melanoma. N. Engl. J. Med. 363(8), 711-723 (2010).

78 Sangro B, Gomez-Martin C, la Mata de M et al. A clinical trial of CTLA-4 blockade with tremelimumab in patients with hepatocellular carcinoma and chronic hepatitis C. J. Hepatol. 59(1), 81-88 (2013).

79 Okazaki T, Honjo T. PD-1 and PD-1 ligands: from discovery to clinical application. Int. Immunol. 19(7), 813-824 (2007).

80 Hamid O, Robert C, Daud A et al. Safety and tumor responses with lambrolizumab (anti-PD-1) in melanoma. N. Engl. J. Med. 369(2), 134-144 (2013).

81 Topalian SL, Hodi FS, Brahmer JR et al. Safety, activity, and immune correlates of anti-PD-1 antibody in cancer. $N$. Engl. J. Med. 366(26), 2443-2454 (2012).

82 Dose escalation study of nivolumab (anti-PD-1; BMS-936558; ONO-4538) in patients (pts) with advanced hepatocellular carcinoma (HCC) with or without chronic viral hepatitis (anti-PD-1 HCC). http://clinicaltrials.gov/show/NCT01658878

83 Zhu AX, Gold PJ, El-Khoueiry AB et al. First-in-man Phase I study of GC33, a novel recombinant humanized antibody against glypican-3, in patients with advanced hepatocellular carcinoma. Clin. Cancer Res. 19(4), 920-928 (2013).

84 A study of RO5137382 (GC33) in patients with advanced or metastatic hepatocellular carcinoma.

http://clinicaltrials.gov/show/NCT01507168

85 Study of GC33 and sorafenib in combination in advanced or metastatic liver cancer (hepatocellular carcinoma). http://clinicaltrials.gov/show/NCT00976170

86 Feng M, Gao W, Wang R et al. Therapeutically targeting glypican-3 via a conformation-specific single-domain antibody in hepatocellular carcinoma. Proc. Natl Acad. Sci. USA 110(12), E1083-E1091 (2013).

87 Castle JC, Kreiter S, Diekmann J et al. Exploiting the mutanome for tumor vaccination. Cancer Res. 72(5), 1081-1091 (2012).

88 Quakkelaar ED, Melief CJM. Experience with synthetic vaccines for cancer and persistent virus infections in nonhuman primates and patients. Adv. Immunol. 114, 77-106 (2012).

89 Rammensee H-G, Singh-Jasuja H. HLA ligandome tumor antigen discovery for personalized vaccine approach. Expert Rev. Vaccines 12(10), 1211-1217 (2013).

90 Mount A, Koernig S, Silva A, Drane D, Maraskovsky E, Morelli AB. Combination of adjuvants: the future of vaccine design. Expert Rev. Vaccines 12(7), 733-746 (2013).

91 Bracci L, Capone I, Moschella F, Proietti E, Belardelli F. Exploiting dendritic cells in the development of cancer vaccines. Expert Rev. Vaccines 12(10), 1195-1210 (2013).

92 Baxevanis CN, Voutsas IF, Tsitsilonis OE. Toll-like receptor agonists: current status and future perspective on their utility as adjuvants in improving anticancer vaccination strategies. Immunotherapy 5(5), 497-511 (2013).

93 Schlom J. Therapeutic cancer vaccines: current status and moving forward. J. Natl Cancer Inst. 104(8), 599-613 (2012).

94 Diken M, Kreiter S, Selmi A, Türeci O, Sahin U. Antitumor vaccination with synthetic mRNA: strategies for in vitro and in vivo preclinical studies. Methods Mol. Biol. 969, 235-246 (2013). 
95 Sandoval F, Terme M, Nizard M et al. Mucosal imprinting of vaccine-induced $\mathrm{CD} 8^{+}$ $T$ cells is crucial to inhibit the growth of mucosal tumors. Sci. Transl. Med. 5(172), 172ra20 (2013).

96 Vonderheide RH, Glennie MJ. Agonistic CD40 antibodies and cancer therapy. Clin. Cancer Res. 19(5), 1035-1043 (2013).

97 Melero I, Hirschhorn-Cymerman D, MoralesKastresana A, Sanmamed MF, Wolchok JD. Agonist antibodies to TNFR molecules that costimulate T and NK cells. Clin. Cancer Res. 19(5), 1044-1053 (2013).

98 Dose escalation study of nivolumab (anti-PD-1; BMS-936558; ONO-4538) in patients (pts) with advanced hepatocellular carcinoma (HCC) with or without chronic viral hepatitis (anti-PD-1 HCC). http://clinicaltrials.gov/show/NCT01658878

99 Anderson AC. Tim-3, a negative regulator of anti-tumor immunity. Curr. Opin. Immunol. 24(2), 213-216 (2012).

100 Sakuishi K, Apetoh L, Sullivan JM, Blazar BR, Kuchroo VK, Anderson AC. Targeting Tim-3 and PD-1 pathways to reverse T cell exhaustion and restore anti-tumor immunity. J. Exp. Med. 207(10), 2187-2194 (2010).

101 Grosso JF, Kelleher CC, Harris TJ et al. LAG-3 regulates CD8 + T cell accumulation and effector function in murine self- and tumor-tolerance systems. J. Clin. Invest. 117(11), 3383-3392 (2007).
102 Woo S-R, Turnis ME, Goldberg MV et al. Immune inhibitory molecules LAG-3 and PD-1 synergistically regulate T-cell function to promote tumoral immune escape. Cancer Res. 72(4), 917-927 (2012).

103 Weinberg AD, Morris NP, KovacsovicsBankowski M, Urba WJ, Curti BD. Science gone translational: the OX40 agonist story. Immunol. Rev. 244(1), 218-231 (2011).

104 Curti BD, Kovacsovics-Bankowski M, Morris $\mathrm{N}$ et al. OX40 is a potent immunestimulating target in late-stage cancer patients. Cancer Res. 73(24), 7189-7198 (2013).

105 Brok den MHMGM, Nierkens S, Figdor CG, Ruers TJM, Adema GJ. Dendritic cells: tools and targets for antitumor vaccination. Expert Rev. Vaccines 4(5), 699-710 (2005).

106 Brok den MHMGM, Sutmuller RPM, Nierkens $S$ et al. Efficient loading of dendritic cells following cryo and radiofrequency ablation in combination with immune modulation induces anti-tumour immunity. Br. J. Cancer 95 (7), 896-905 (2006).

107 Sellge G, Lorentz A, Gebhardt T et al. Human intestinal fibroblasts prevent apoptosis in human intestinal mast cells by a mechanism independent of stem cell factor, IL-3, IL-4, and nerve growth factor. J. Immunol. 172(1), 260-267 (2004).

108 Wissniowski TT, Hänsler J, Neureiter D et al. Activation of tumor-specific
T lymphocytes by radio-frequency ablation of the VX2 hepatoma in rabbits. Cancer Res. 63(19), 6496-6500 (2003).

109 Geissler M, Mohr L, Weth R et al. Immunotherapy directed against alphafetoprotein results in autoimmune liver disease during liver regeneration in mice. Gastroenterology 121(4), 931-939 (2001).

110 Hanke P, Serwe M, Dombrowski F, Sauerbruch T, Caselmann WH. DNA vaccination with AFP-encoding plasmid DNA prevents growth of subcutaneous AFP-expressing tumors and does not interfere with liver regeneration in mice. Cancer Gene Ther. 9(4), 346-355 (2002).

111 Melero I, Grimaldi AM, Perez-Gracia JL, Ascierto PA. Clinical development of immunostimulatory monoclonal antibodies and opportunities for combination. Clin. Cancer Res. 19(5), 997-1008 (2013).

112 Wolchok JD, Kluger H, Callahan MK et al. Nivolumab plus Ipilimumab in advanced melanoma. N. Engl. J. Med. 369(2), 122-133 (2013).

113 Morales-Kastresana A, Sanmamed MF, Rodriguez I et al. Combined immunostimulatory monoclonal antibodies extend survival in an aggressive transgenic hepatocellular carcinoma mouse model. Clin. Cancer Res. 19(22), 6151-6162 (2013). 